\title{
Drug reaction with eosinophilia and systemic syndrome: Systemic drug reaction
}

\section{Zubin Dev Sharma, Vijayashree Shrirang Gokhale, Sunit Lokwani, Arjun Lal Kakrani}

Department of Medicine, Pad. Dr. D.Y.Patil Medical College and Research Hospital, Sant Tukaram

Nagar, Pimpri, Pune, India

Address for the Correspondence:

Dr. Zubin Sharma,

Department of Medicine,

Pad. Dr. D. Y. Patil Medical College and Research Hospital, Sant Tukaram Nagar, Pimpri, Pune - 18, India.

E-mail: drzubinsharma@ gmail.com

\begin{tabular}{|l|}
\hline Access this article online \\
\hline Website: www.ijmedph.org \\
\hline DOI: 10.4103/2230-8598.109328 \\
\hline Quick response code: \\
\hline
\end{tabular}

Drug reaction with eosinophilia and systemic (DRESS) syndrome is a severe systemic drug reaction with a reported mortality of $10 \%{ }^{5}$. The estimated incidence of this syndrome ranges from 1 in 1000 to 1 in 10,000 drug exposures. The syndrome consists of a severe skin eruption, fever, hematologic abnormalities (eosinophilia or atypical lymphocytes), and internal organ involvement. Here, we report a case of DRESS syndrome in a young male who presented with acute hepatitis.

Key words: Drug reaction with eosinophilia and systemic syndrome, drug reaction, hepatitis leflunomide, skin rash

\section{INTRODUCTION}

Drug reaction with eosinophilia and systemic (DRESS) syndrome is a potentially lethal drug reaction that occurs because of commonly used drugs like anticonvulsants and NSAIDS. Its features include severe skin rash associated with eosinophilia and systemic signs. Most common organ involved is the liver, leading to acute hepatitis-like condition. The other note-worthy features are a delayed onset, usually 2-6 weeks after the initiation of drug therapy, and the possible persistence or aggravation of symptoms despite the discontinuation of the culprit drug. It is also known as drug-induced hypersensitivity syndrome (DIHS). This case report highlights this syndrome and reflects the fact that all physicians and public health workers should be aware of this condition and need to identify this early in the form of rash, lymphadenopathy, and systemic signs.

\section{CASE REPORT}

A 22-year-old male patient presented with complaints of fever with chills and rigors of 7 days duration associated with an erythematous maculopapular rash all over body with itching. The patient gave no history of loose motions, cough, sore throat, headache, myalgia/arthalgia, mosquito bite, tick bite, or high-risk sexual behavior. The patient gave history of bilateral ankle swelling since 2 months prior to this presentation for which he was taking leflunamide and NSAIDS in the form of tablet diclofenac $(50 \mathrm{mg})$ twice daily. The patient had stopped diclofenac after 2 days but continued with leflunamide. Patient was nonalcoholic and no history of any substance abuse. The patient was febrile, with an axillary temperature of 102 degrees F. There was cervical lympadenopathy, soft, around 2-4 cms, mildly tender and mild swelling at ankle joint.

There was an erythematous maculopapular rash seen all over the body, with exfoliation over the face. The mucous membranes were spared [Figures 1 and 2].

A complete hemogram showed hemoglobin of $12 \mathrm{gm} \%$ with total leukocyte count of $12000 /$ cumm. The differential count showed 50\% neutrophils, $28 \%$ lymphocytes, and $20 \%$ eosinophils, with absolute eosinophil count of 2400 cells/cumm. The platelet count was 1.2 lakhs/cumm.

The liver function tests on first day showed normal bilirubin levels with serum alanine transferase (ALT) of $157 \mathrm{IU} / 1$ and serum alkaline phosphtase (ALP) of $146 \mathrm{IU} / \mathrm{l}$. Renal function tests and serum electrolytes were normal. An abdominal ultrasound showed bright echotexture of liver.

On second day, the liver function tests were repeated and it showed an increase in ALT to 416 IU/1 and ALP to $207 \mathrm{IU} / \mathrm{l}$. The patients was negative for hepatitis A, B, C, E antibodies, HIV, dengue NS1 antigen, rapid malaria, Paul Bunnel, Weil Felix test, ASO titers, RA factor, Anti CCP, and HLA B27 markers. 


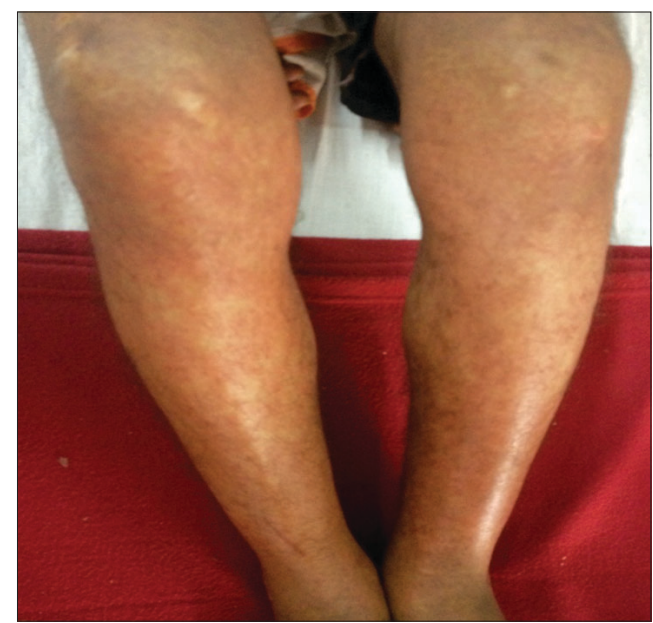

Figure 1: Severe rash on legs

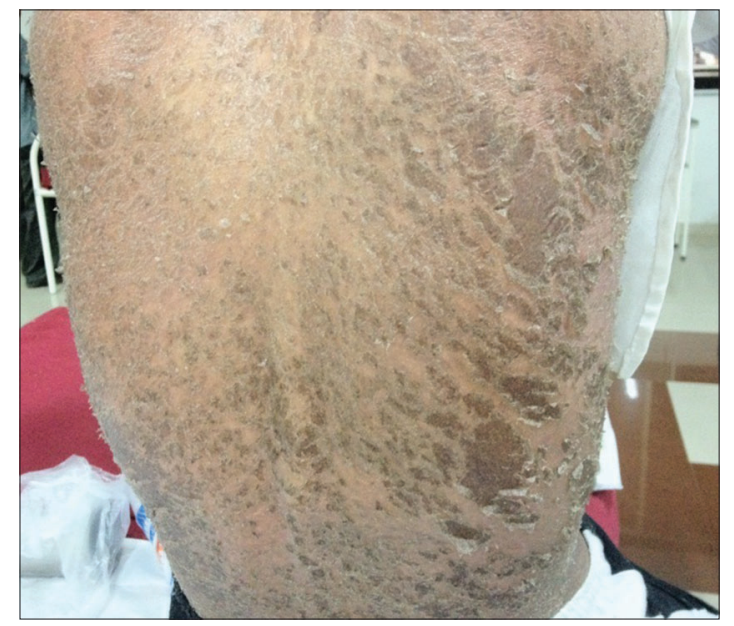

Figure 2: Severe exfoliation after few days

The peripheral smear showed atypical lymphocytes. A bone marrow biopsy was performed, which was essentially normal. Urine examination and stool examination were normal.

Liver function tests continued to deteriorate further and reached a maximum of serum bilirubin of total 5.5 with serum ALT of 1190 IU/1. [Table 1] Blood and urine culture revealed no growth.

The exfoliation increased to involve the whole body by around $15^{\text {th }}$ day of admission. Patient was started on oral prednisolone $40 \mathrm{mg}$ daily with multivitamin supplements and antihistaminics and liquid paraffin for topical use. The rash started to decrease and the liver function tests also showed improvement after $15^{\text {th }}$. The patient was discharged on $25^{\text {th }}$ day of admission with a final diagnosis of DRESS possibly due to leflunamide use or NSAIDS [Table 1].

\section{DISCUSSION}

The DRESS is a severe adverse drug-induced reaction. The estimated incidence of this syndrome ranges from 1 in 1000 to 1 in 10,000 drug exposures. ${ }^{[1]}$ The acronym designated by Bocquet et al. ${ }^{[2]}$ described a severe skin eruption, fever, hematologic

\begin{tabular}{lccccc} 
Table 1: Sequential rise in liver function tests & LFTs \\
\hline Test/day & On admission & Day 2 & Day 3 & Day $\mathbf{7}$ & Day 25 \\
\hline S. Bilirubin (T) mg/dl & 1.1 & 2.8 & 4.5 & 5.5 & 1.2 \\
ALT (IU/I) & 157 & 416 & 580 & 1190 & 76 \\
ALP (IU/I) & 146 & 207 & 217 & 214 & 102 \\
\hline
\end{tabular}

$\mathrm{ALT}=$ Alanine transferase, $\mathrm{ALP}=$ Alkaline phosphtase

abnormalities (eosinophilia or atypical lymphocytes), and internal organ involvement. It has been also been called as Dapson syndrome or anticonvulsant hypersensitivity syndrome. The pathogenesis includes detoxification defects leading to reactive metabolite formation and subsequent immunological reactions, slow acetylation, and reactivation of human herpes, including Epstein-Barr virus and human herpesvirus (HHV)-6 and HHV-7. The detection of HHV-6 reactivation has even been recently proposed as a diagnostic marker for DRESS. ${ }^{[3]}$

This type of reaction is most commonly seen with the use of the following seven different drug groups:

i. anticonvulsants, such as the aromatic anticonvulsants (phenytoin, carbamazepine, phenobarbital, and primidone), mexiletine, lamotrigine, valproate, ethosuximide, and zonisamide;

ii. antidepressants (desipramine, amitriptyline, and fluoxetine);

iii. sulfonamides and sulfones (dapsone, sulfasalazine, trimethoprim-sulfamethoxazole, and salozosulphopyridine);

iv. anti-inflammatory drugs (piroxicam, naproxen, diclofenac, sundilac, phenylbutazone, and ibuprofen);

v. anti-infectives (abacavir, cidofovir, terbinafine, nevirapine, minocycline, linezolid, doxycycline, telaprevir, nitrofurantoin, zalci-tabine, spiramycin, metronidazole, piperacillintazo-bactam, and ceftriaxone);

vi. angiotensin-converting enzyme inhibitors (captopril and enalapril);

vii. beta-blockers (atenolol and celiprolol). Cases have been reported with allopurinol, gold salts, thalidomide, calcium channel blockers (diltiazem), ranitidine, sorbinil, azathioprine, dobutamine, methimazole, leflunomide, propylthiouracil, and efalizumab.

There is no gold standard for diagnosis, and at least two diagnostic criteria have been proposed, the regiSCAR Criteria and the Japanese Consensus Group Criteria. ${ }^{[4]}$

A similar case was reported by Vaish, et al. ${ }^{[5,6]}$ in which the authors reported a case of DRESS syndrome with severe exfoliative dermatitis with acute hepatitis associated with intake of leflunomide.

Thus, this case shows the occurrence of DRESS syndrome in relation to possible use of leflunomide and shows that early diagnosis is imperative for initiation of treatment in this severe drug reaction.

Thus, diagnosis of DRESS should be suspected with presence of skin rash, liver involvement, fever, hypereosinophilia, and lymphadenopathy. DRESS/DIHS is a serious illness with a mortality rate of close to $10 \%{ }^{[6]}$ 


\section{REFERENCES}

1. Fiszenson-Albala F, Auzerie V, Mahe E, Farinotti R, Durand-Stocco C, Crickx B, et al. A 6-month prospective survey of cutaneous drug reactions in a hospital setting. Br J Dermatol 2003;149:1018-22.

2. Bocquet H, Bagot M, Roujeau JC. Drug-induced pseudolymphoma and drug hypersensitivity syndrome (Drug Rash with Eosinophilia and Systemic Symptoms: DRESS). Semin Cutan Med Surg 1996;15:250-7.

3. Roujeau JC, Stern RS. Severe adverse cutaneous reactions to drugs. N Engl J Med 1994;331:1272-85.

4. Chaiken BH, Goldberg BI, Segal JP. Dilantin hypersensitivity: Report of a case of hepatitis with jaundice, pyrexia, and exfoliative dermatitis. N Engl J Med 1950;242:897-8.
5. Criado PR, Criado PF, Avancini JM, Santi C. Drug Reaction with Eosinophilia and Systemic Symptoms (DRESS)/Drug-Induced Hypersensitivity Syndrome (DIHS): A review of current concepts. An Bras Dermatol 2012;87:435-49.

6. Vaish AK, Tripathi AK, Gupta LK, Jain N, Agarwal A, Verma SK. An unusual case of DRESS syndrome due to leflunomide. BMJ Case Rep 2011.

How to cite this article: Sharma ZD, Gokhale VS, Lokwani S, Kakrani AL. Drug reaction with eosinophilia and systemic syndrome: Systemic drug reaction. Int J Med Public Health 2013;3:69-71.

Source of Support: Nil, Conflict of Interest: None declared. 